\title{
Inverse Scattering for the Stationary Wave Equation with a Friction Term in Two Dimensions
}

\author{
Dedicated to Professor Hiroshi Isozaki on his sixtieth birthday
}

by

Michiyuki Watanabe

\begin{abstract}
We consider the inverse scattering problem for the two-dimensional stationary wave equation with a friction term. We prove that the friction coefficient can be uniquely reconstructed from the scattering amplitude at a fixed low energy. A reconstruction procedure is also given. The method is to reduce the inverse scattering problem to an inverse boundary value problem. We use the $\bar{\partial}$-method for a $3 \times 3$ first order elliptic system to give the reconstruction procedure.
\end{abstract}

2010 Mathematics Subject Classification: Primary 35R30; Secondary 35P25.

Keywords: inverse scattering, inverse boundary value problem, Schrödinger equations.

\section{$\S 1$. Introduction}

\section{§1.1. Problems and results}

Let $b(x)$ be a real-valued continuous function which decays sufficiently fast at infinity. We consider the wave equation with friction coefficient $b(x)$ :

$$
w_{t t}(t, x)-\Delta w(t, x)+b(x) w_{t}(t, x)=0, \quad(t, x) \in \mathbb{R} \times \mathbb{R}^{n} .
$$

The equation (1.1) is regarded as a perturbation of the free wave equation

$$
w_{0 t t}(t, x)-\Delta w_{0}(t, x)=0, \quad(t, x) \in \mathbb{R} \times \mathbb{R}^{n} .
$$

Then we can consider the scattering problem and the inverse scattering problem of identifying the friction coefficient $b(x)$. There are some works on these problems for

Communicated by H. Okamoto. Received January 26, 2011. Revised February 16, 2012, and June $11,2012$.

M. Watanabe: Mathematical and Natural Sciences, Faculty of Education, Niigata University, 8050 Ikarashi 2-no-cho, Nishi-ku, Niigata, 950-2181, Japan;

e-mail: mwatanab@ed.niigata-u.ac.jp

(C) 2013 Research Institute for Mathematical Sciences, Kyoto University. All rights reserved. 
the wave equation (1.1). In the multi-dimensional case $n \geq 3$, Mochizuki ([?]-[20]) proved the existence and completeness of wave operators for (1.1) with small $b(x)$. He also gave an expression for the scattering amplitude and gave a reconstruction procedure of small $b(x)$ from the scattering amplitude at a fixed energy. For the scattering problem, we also refer the reader to [24].

In two dimensions, Nakazawa [25] studied the scattering problem for the wave equation (1.1) with small dissipative terms. Recently, Kadowaki, Nakazawa and Watanabe [13] improved the smallness assumption on the dissipative terms.

As reviewed so far, smallness assumptions on $b(x)$ have been required in the scattering and the inverse scattering problem. For the problem of two-dimensional inverse scattering at a fixed energy, while a uniqueness theorem was proved in [30], there has not been much research on the reconstruction problem of $b(x)$. Here, the uniqueness problem is whether the scattering amplitude uniquely determines the friction coefficient $b(x)$, and the reconstruction problem is whether $b(x)$ can be calculated in terms of the scattering amplitude.

The purpose of this study is to give an answer to the reconstruction problem in two dimensions.

Consider the stationary wave equation of (1.1). The substitution $w=e^{i \sqrt{E} t} u(x)$ in (1.1) yields

$$
-\Delta u+i \sqrt{E} b(x) u=E u \quad \text { in } \mathbb{R}^{2} .
$$

To begin with, we define the scattering amplitude via outgoing eigenfunctions. For $s \in \mathbb{R}$, we consider the weighted $L^{2}$ space

$$
L^{2, s}\left(\mathbb{R}^{n}\right)=\left\{u:\left(\int_{\mathbb{R}^{n}}\left|(1+|x|)^{s} u(x)\right|^{2} d x\right)^{1 / 2}<\infty\right\} .
$$

Theorem 1.1 ([12]). Assume that $b(x)$ is a complex-valued $C_{0}^{\infty}\left(\mathbb{R}^{2}\right)$ function. Then there is a discrete set $\mathcal{E}_{0}$ in a neighborhood of $(0, \infty)$ such that for $E \in$ $(0, \infty) \backslash \mathcal{E}_{0}$ and $\omega \in S^{1}$, there exists a unique solution $u(x, E, \omega)$ of $(1.2)$ with the radiation condition

$$
\left(\frac{\partial}{\partial r}-i \sqrt{E}\right) \psi \in L^{2,-\alpha}\left(\mathbb{R}^{2}\right), \quad 0<\alpha<1 / 2,
$$

where $r=|x|$ and $\psi=u-e^{i \sqrt{E} \omega \cdot x}$.

The solution $u$ has the asymptotic expansion

$$
u(x, E, \omega)=e^{i \sqrt{E} \omega \cdot x}+\frac{e^{i \sqrt{E}|x|}}{|x|^{1 / 2}} A(E, \theta, \omega)+o\left(|x|^{-1 / 2}\right), \quad \theta=x /|x|, \quad|x| \rightarrow \infty .
$$


The first term of the right-hand side is an incident plane wave. The second term is a spherical wave, termed the scattered wave, since it is caused by the frictional force of the medium. Theorem 1.1 and the asymptotic expansion (1.3) show that the scattered wave exists and for large value of $|x|$, where the scattered wave is to be measured, the solution $u$ to (1.2) has the asymptotic form (1.3).

The amplitude of the scattered wave is called the scattering amplitude, and is represented by

$$
A(E, \theta, \omega)=-\frac{1}{4} \sqrt{\frac{2}{\pi}} E^{1 / 4} e^{-i \pi / 4} \int_{\mathbb{R}^{2}} e^{-i \sqrt{E} \theta \cdot x} b(x) u(x) d x,
$$

where $u$ is the solution given in Theorem 1.1 .

In this paper we consider the inverse scattering problem of whether the frictional force of the medium is uniquely determined from observations of the amplitude of the scattered wave. The mathematical formulation of this problem is as follows: Determine $b(x)$ from $A(E, \theta, \omega)$.

The following statement shows that if the friction coefficient has compact support and is bounded by a constant, then for any sufficiently low energy level, the coefficient can be uniquely reconstructed from the scattering amplitude at a fixed energy. This result does not require the smallness assumption on the friction coefficient, which means that global uniqueness holds for low energy levels.

Let $\Omega=B_{R}=\left\{x \in \mathbb{R}^{2}:|x| \leq R\right\}$ and let $W^{m, p}(\Omega)$ denote the usual $L^{p}$ Sobolev space of order $m$. In what follows, $C(a, b, c)$ always means a positive constant, possibly different in different occurrences, depending on $a, b, c$.

Theorem 1.2. Assume that $b(x)$ is a complex-valued $C_{0}^{\infty}\left(\mathbb{R}^{2}\right)$ function and that $\operatorname{supp} b \subset \Omega$. Suppose that for some $p>2$ the $W^{1, p}(\Omega)$ norm of $b(x)$ is bounded by a constant $M$ :

$$
\|b\|_{W^{1, p}(\Omega)} \leq M .
$$

Then there exists a constant $N=N(p, \Omega, M)$ such that $b(x)$ can be uniquely determined from the corresponding scattering amplitude $A(E, \theta, \omega)$ at a fixed $E \in$ $(0, N) \backslash \mathcal{E}_{0}$. Moreover, we give a reconstruction procedure to identify $b(x)$ from $A(E, \theta, \omega)$.

In order to prove Theorem 1.2, we reduce the inverse scattering problem to an inverse boundary value problem. Put $V(x)=\sqrt{E}(i b(x)-\sqrt{E})$. Then we can rewrite the equation (1.2) as

$$
-\Delta u+V u=0 \quad \text { in } \Omega .
$$

In general, assume that $V(x)$ is a complex-valued function in $L^{p}(\Omega), p>2$, and suppose that 0 is not a Dirichlet eigenvalue for the operator $-\Delta+V$ in $\Omega$. One can 
then show that for any $f \in C^{1, \alpha}(\partial \Omega)$ there exists a unique solution $u \in C^{1, \alpha}(\bar{\Omega})$ of (1.5) with $\left.u\right|_{\partial \Omega}=f$. Here $C^{1, \alpha}(\Omega)$ is the usual Hölder space and $\alpha=(p-2) / p$ $(p>2)$. Therefore, we can define the Dirichlet-to-Neumann map (DN map) $\Lambda_{V}$ : $C^{1, \alpha}(\partial \Omega) \rightarrow C^{\alpha}(\partial \Omega)$ by

$$
\Lambda_{V}:\left.f \mapsto \frac{\partial u}{\partial \nu}\right|_{\partial \Omega}
$$

where $\nu$ denotes the unit outer normal to $\partial \Omega$.

The inverse boundary value problem is to identify the complex-valued function $V(x)$ from the DN map $\Lambda_{V}$. In particular, the uniqueness problem is whether the DN map uniquely determines $V(x)$. When the uniqueness holds, we want to calculate $V(x)$ in terms of the DN map. We call it the reconstruction problem.

As is well known, for the Schrödinger equation with compact support potentials, the scattering amplitude with a fixed energy uniquely determines the DN map $\Lambda_{V}$ (see, e.g., Nachman [21]). Therefore, it is enough to show that the complex coefficient $V(x)$ in (1.5) can be uniquely reconstructed from $\Lambda_{V}$.

There are many works on the inverse boundary value problem for (1.5). In the multi-dimensional case $(n \geq 3)$, the global uniqueness was proved by Sylvester and Uhlmann [28]. Nachman [23] gave a solution of the reconstruction problem. The algorithm of Nachman [23] was derived independently by Novikov [26]. For details on the inverse boundary value problem, we also refer the reader to $[9]$.

In two dimensions, the local uniqueness for complex-valued potentials with small $L^{p}(\Omega)$ norm was proved by Kang [14] and Kang and Uhlmann [15]. Bukhgeim [3] resolved the global uniqueness problem for complex-valued $V \in L^{p}(\Omega), p>2$. He also showed that smooth potentials can be reconstructed from boundary measurements with a special boundary condition. It is still not clear whether we can reconstruct a complex-valued potential in terms of the DN map. A recent result of Imanuvilov, Uhlmann and Yamamoto [8] gives the global uniqueness of a complexvalued potential from Cauchy data measured on part of the boundary.

On the reconstruction problem in two dimensions, there are some partial results. Isakov and Nachman [10] gave a uniqueness theorem and a reconstruction procedure for non-negative function $V(x) \geq 0$ in $L^{p}(\Omega), p>1$. Isakov and Sun [11] proved that a real-valued potential $V \in C^{2, \alpha}\left(\mathbb{R}^{2}\right)$ with compact support can be uniquely determined from the associated scattering amplitude at finitely many energies. For other results on the inverse scattering problem at a fixed energy, we refer to [6], [27].

We should mention that the global uniqueness and reconstruction procedure for the conductivity potential $V=\gamma^{-1 / 2} \Delta \gamma^{1 / 2}$ have been established. Nachman [22] proved uniqueness and gave a reconstruction procedure for $\gamma \in W^{2, p}(\Omega)$, 
$p>1$, with a positive lower bound. Brown and Uhlmann [2] improved the regularity assumption and showed uniqueness for $\gamma \in W^{1, p}(\Omega), p>2$. A reconstruction procedure for $\gamma \in W^{1+\varepsilon, p}(\Omega)(p>2, \varepsilon>0)$ was given by Knudsen and Tamasan [17]. For $\gamma \in L^{\infty}(\Omega)$, the global uniqueness and reconstruction problem was resolved by Astala and Päivärinta [1]. Cheng and Yamamoto [4] proved the global uniqueness for real-valued coefficients of an elliptic equation which contains the conductivity equation. Francini [5] proved the uniqueness for the complex conductivity $\gamma \in W^{1, \infty}(\Omega)$ with a smallness condition on the imaginary part of $\gamma$.

As reviewed so far, solutions of the reconstruction problem in two dimensions have been studied for real-valued potentials or conductivity type potential. However, there have been few results on the reconstruction problem for complex-valued potentials.

In this paper we give a partial answer to the reconstruction problem for complex-valued potentials in two dimensions.

Theorem 1.3. Assume that $V$ is a complex-valued $W^{1, p}(\Omega)$ function for some $p>2$. Then there exists a positive constant $M=M(p, \Omega)$ such that if $\|V\|_{W^{1, p}(\Omega)}$ $\leq M$, there is a reconstruction procedure to identify $V(x)$ for any $x \in \bar{\Omega}$ from $\Lambda_{V}$.

Assuming Theorem 1.3, we prove Theorem 1.2 as follows. Put $V(x)=$ $\sqrt{E}(i b(x)-\sqrt{E})$. Then $\|V\|_{W^{1, p}(\Omega)}$ is small when $E$ is small. By the reduction argument in Nachman [21], $\Lambda_{V}$ can be calculated from $A(E, \theta, \omega)$. It follows from Theorem 1.3 that $V(x)$ can be uniquely reconstructed from $A(E, \theta, \omega)$. Thus we obtain $b(x)$ from

$$
b(x)=\frac{V(x)+E}{\sqrt{E} i} .
$$

The proof of Theorem 1.3 relies on the $\bar{\partial}$-method for a first order elliptic system. In the $\bar{\partial}$-method given by Nachman [22], symmetry of solutions plays an important role. A difficulty in the complex-valued case is the lack of symmetry of solutions. This can be removed by considering the system

$$
\left\{\left(\begin{array}{ccc}
\bar{\partial}_{z} & 0 & 0 \\
0 & \partial_{z} & 0 \\
0 & 0 & \partial_{z}
\end{array}\right)-\left(\begin{array}{ccc}
0 & q & 0 \\
1 & 0 & 0 \\
0 & q & 0
\end{array}\right)\right\} \Phi(z)=0, \quad q=q(z)=V(z) / 4
$$

where we identify $x=\left(x_{1}, x_{2}\right) \in \mathbb{R}^{2}$ with the complex variable $z=x_{1}+i x_{2}$ and use the complex notations

$$
\partial_{z}=\frac{1}{2}\left(\partial_{x_{1}}-i \partial_{x_{2}}\right), \quad \bar{\partial}_{z}=\frac{1}{2}\left(\partial_{x_{1}}+i \partial_{x_{2}}\right)
$$


Consequently, we give a complete characterization of the trace of the complex geometrical optics solutions ( $C G O$ solutions). This characterization provides a method for finding the trace of the CGO solution from $\Lambda_{V}$ (see Lemma 4.2 below). The characterization in Lemma 4.2 is the main ingredient in the reconstruction procedure. It will also be shown that the properties of the CGO solution to (1.6) give a new reconstruction procedure.

\section{$\S 1.2$. Reconstruction procedure}

We shall summarize our reconstruction procedure of $b(x)$ from the corresponding scattering amplitude $A(E, \theta, \omega)$. In order to do this we adopt some notations.

- $z_{R}$ is the real part of $z$ and $z_{I}$ is the imaginary part of $z$.

- $e_{k}=e_{k}(z)=e^{i(k z+\bar{k} \bar{z})}$.

- $\nu=\left(\nu_{1}, \nu_{2}\right)$ is the unit outer normal to $\partial \Omega ; \eta=\nu_{1}+i \nu_{2} ; \tau=\left(-\nu_{2}, \nu_{1}\right)$. The tangential derivative on $\partial \Omega$ is denoted by

$$
\frac{\partial}{\partial \tau}=\tau \cdot \nabla
$$

- Let $\rho(s)$ be an arc length parameterization of $\partial \Omega$. We define an operator $\mathcal{L}$ by

$$
(\mathcal{L} f)(\rho(s))=\int_{0}^{s} f(\rho(t)) d t
$$

- Let $\mathcal{F}$ be the Cauchy integral,

$$
(\mathcal{F} g)(z)=\frac{1}{2 \pi i} \int_{\partial \Omega} \frac{g(\zeta)}{\zeta-z} d \zeta
$$

The conjugate of $\mathcal{F}$ is denoted by $\overline{\mathcal{F}}$, i.e., $(\overline{\mathcal{F}} g)(z):=\overline{(\mathcal{F} g)}(z)$. We then define an operator $\mathcal{F}$ by

$$
\mathcal{F} \boldsymbol{g}=\left(\begin{array}{ccc}
\mathcal{F} & 0 & 0 \\
0 & \overline{\mathcal{F}} & 0 \\
0 & 0 & \overline{\mathcal{F}}
\end{array}\right)\left(\begin{array}{l}
g_{1} \\
g_{2} \\
g_{3}
\end{array}\right) .
$$

- Let $\boldsymbol{X}=\left(x_{i j}\right)$ be a $3 \times 3$ matrix. We define an operator $\mathcal{P}_{k}$ by

$$
\mathcal{P}_{k} \boldsymbol{X}=\left(\begin{array}{ccc}
x_{11} & e_{-\bar{k}} x_{12} & e_{-\bar{k}} x_{13} \\
e_{k} x_{21} & x_{22} & x_{23} \\
e_{k} x_{31} & x_{32} & x_{33}
\end{array}\right) .
$$

- $\boldsymbol{E}=\boldsymbol{E}(z, k)=\left(\begin{array}{ccc}e^{i k z} & 0 & 0 \\ 0 & e^{-i k \bar{z}} & 0 \\ 0 & 0 & e^{-i k \bar{z}}\end{array}\right)$. 
- The $j$-th column vector of the matrix $\boldsymbol{X}=\left(x_{i j}\right)$ is denoted by $\boldsymbol{x}^{(j)}$.

- $\boldsymbol{I}$ is the unit matrix and $\boldsymbol{O}$ is the zero matrix.

The reconstruction procedure for the friction coefficient $b(x)$ now consists of several steps.

Step 1. Calculate the DN map $\Lambda_{V}$ from the scattering amplitude $A(E, \theta, \omega)$ at a fixed $E>0$ for all $\theta, \omega \in S^{1}$ (see, e.g., Isakov and Nachman [10], Nachman ([21], $[23$, p. 567]) and Ikehata [7, pp. 38-44]).

Step 2. Let $k \in \mathbb{C}$. Solve the system of equations on $\partial \Omega$

$$
\left\{\begin{array}{l}
\left.\mathcal{P}_{-k} \mathcal{F}\left(\mathcal{P}_{k} \boldsymbol{\Psi} \boldsymbol{E}^{-1}\right)\right|_{\partial \Omega}=\boldsymbol{I} \\
\left(\begin{array}{ccc}
i \Lambda_{V} \mathcal{L} & 0 & -i \Lambda_{V} \mathcal{L} \\
0 & i \frac{\partial}{\partial \tau} & 0
\end{array}\right)\left(\begin{array}{lll}
\eta & 0 & 0 \\
0 & 1 & 0 \\
0 & 0 & \bar{\eta}
\end{array}\right) \boldsymbol{\Psi}=\left(\begin{array}{ccc}
\eta & 0 & \bar{\eta} \\
-\eta & 0 & \bar{\eta}
\end{array}\right) \boldsymbol{\Psi}
\end{array}\right.
$$

to determine the $3 \times 3$ matrix $\boldsymbol{\Psi}(\cdot, k)=\left(\psi_{i j}\right)$ on $\partial \Omega$ such that $\boldsymbol{\psi}^{(j)} \in C^{\alpha}(\partial \Omega) \times$ $C^{1, \alpha}(\partial \Omega) \times C^{\alpha}(\partial \Omega), j=1,2,3$, where $\alpha=(p-2) / p$ for some $p>2$ (see Lemma 4.2 below).

Step 3. Define

$$
\boldsymbol{S}(k)=\left(\begin{array}{cc}
0 & \frac{1}{2 \pi i} \int_{\partial \Omega} e^{-i \bar{k} \zeta} \psi_{12}(\zeta, k) d \zeta \\
-\frac{1}{2 \pi i} \int_{\partial \Omega} e^{i \bar{k} \bar{\zeta}} \psi_{21}(\zeta, k) d \bar{\zeta} & 0
\end{array}\right)
$$

Let $\bar{\partial}_{k}^{-1}$ be the integral operator

$$
\bar{\partial}_{k}^{-1} f(k)=-\frac{1}{\pi} \int_{\mathbb{R}^{2}} \frac{f(\zeta)}{\bar{\zeta}-\bar{k}} d \zeta_{R} d \zeta_{I}
$$

Introduce the matrix

$$
\boldsymbol{\Gamma}_{z}(k)=\left(\begin{array}{cc}
e_{\bar{k}}(z) & 0 \\
0 & e_{-k}(z)
\end{array}\right)
$$

Solve the integral equation

$$
\boldsymbol{M}(z, k)=\boldsymbol{I}+\bar{\partial}_{k}^{-1}\left(\boldsymbol{M}(z, \cdot) \boldsymbol{\Gamma}_{z} \boldsymbol{S}\right)(k)
$$

for the $2 \times 2$ matrix $\boldsymbol{M}(z, \cdot), z \in \Omega$, with the condition $\boldsymbol{M}(z, \cdot)-\boldsymbol{I} \in L^{2, \delta}(\mathbb{C})$ for $-1<\delta<0$ (see Kang and Uhlmann [15]).

Step 4. Define $\varphi(z, k)=e^{-i k \bar{z}} m_{12}(z, k)$ and set

$$
V(z)=\frac{4}{\pi} \lim _{\left|k_{0}\right| \rightarrow \infty} \int_{\left|k-k_{0}\right|<1} e^{i k \bar{z}} \bar{\partial}_{z} \varphi(z, k) d k_{R} d k_{I} .
$$


Then $b(x)$ is obtained from the formula

$$
b(x)=\frac{V(z)+E}{\sqrt{E} i}
$$

(see Brown and Uhlmann [2, pp. 1024-1025]).

The properties of the third row of the $3 \times 3$ matrix $\boldsymbol{\Phi}$, the CGO solution to (1.6), turn out to give us another reconstruction procedure.

First, according to the above procedure (Steps 1 and 2), we determine functions $\psi_{11}, \psi_{12}, \psi_{21}$. Then we perform Steps $3^{\prime}$ and $4^{\prime}$ below.

Step $3^{\prime}$. Let $\boldsymbol{S}(k)=\left(s_{i j}(k)\right)$ and $\boldsymbol{\Gamma}_{z}(k)$ be the $2 \times 2$ matrix given in Step 3 . Set

$$
\begin{aligned}
& \boldsymbol{S}_{0}(k)={ }^{t} \boldsymbol{S}(k), \\
& \boldsymbol{S}_{1}(k)=\left(\begin{array}{c}
\frac{1}{2 \pi i} \int_{\partial \Omega} e^{i \bar{k} \bar{\zeta}} \psi_{11}(\zeta, k) d \zeta-i \bar{k} s_{21}(k) \\
0
\end{array}\right) .
\end{aligned}
$$

Solve the integral equation

$$
\boldsymbol{m}(z, k)=\boldsymbol{I}+\bar{\partial}_{k}^{-1}\left(\boldsymbol{\Gamma}_{z}^{*} \boldsymbol{S}_{0} \boldsymbol{m}(z, \cdot)-i \boldsymbol{S}_{1}\right)(k)
$$

for $\boldsymbol{m}(z, k)={ }^{t}\left(m_{1}(z, k), m_{2}(z, k)\right), z \in \Omega$, with the condition $\boldsymbol{m}(z, \cdot)-\boldsymbol{e}_{1} \in$ $L^{2, \delta}(\mathbb{C})$ for $-1<\delta<0$, where $\boldsymbol{\Gamma}_{z}^{*}(k)=\boldsymbol{\Gamma}_{z}(-\bar{k})$ and $\boldsymbol{e}_{1}={ }^{t}(1,0)$.

Step $4^{\prime}$. Set

$$
V(z)=4 \partial_{z} \lim _{|k| \rightarrow \infty} m_{2}(z, k), \quad z \in \Omega .
$$

Then $b(x)$ is obtained from

$$
b(x)=\frac{V(z)+E}{\sqrt{E} i} .
$$

Remark 1.4. Steps $2,3^{\prime}$ and $4^{\prime}$ are new in the case where the potential is complex-valued.

Remark 1.5. We prove Theorem 1.3 without using an extension argument involving $x \in \mathbb{R}^{2}$ instead of $x \in \Omega$. In order to derive the boundary integral equation to associate the boundary value of the CGO solution with the DN map, it is the standard argument to employ the jump formula on the boundary:

$$
\lim _{\varepsilon \rightarrow 0} \mathcal{S} f(z \pm \nu \varepsilon)=\mp \frac{1}{2} f(z)+\frac{1}{2 \pi i} \text { p.v. } \int_{\partial \Omega} \frac{f(\zeta)}{\zeta-z} d \zeta, \quad z \in \partial \Omega,
$$

where $\mathcal{S}$ is the Cauchy integral operator

$$
\mathcal{S} f(z)=\frac{1}{2 \pi i} \int_{\partial \Omega} \frac{f(\zeta)}{\zeta-z} d \zeta, \quad z \in \mathbb{R}^{2} \backslash \partial \Omega
$$


(see, e.g., Knudsen [16, pp. 34-48]). The argument based on the jump formula shows that the CGO solution should be constructed in $\mathbb{R}^{2}$.

Instead of the jump formula, we apply the generalized Cauchy integral formula to derive the boundary equation given in Step 2. Our method shows that it is enough to construct the CGO solution in $\Omega$. Consequently, we can prove Theorem 1.3 by an argument in $\Omega$ which does not use an extension to $\mathbb{R}^{2}$.

The following is the outline of the remaining sections of this paper.

Section 2. Summarizes some useful lemmas.

Section 3. Presents a construction of the CGO solution and its properties.

Section 4. Discusses the determination of the boundary value of the CGO solution from the DN map.

Section 5. Provides the unique solvability of the $\bar{\partial}$-equation and the proof of Theorem 1.3.

\section{§2. Preliminaries}

In this section we state some useful lemmas. Let $T$ and $\bar{T}$ denote the Cauchy type operators

$$
T f(z) \equiv T_{\Omega} f(z)=-\frac{1}{\pi} \int_{\Omega} \frac{f(\zeta)}{\zeta-z} d \zeta_{R} d \zeta_{I}, \quad \bar{T} f(z)=\overline{T \bar{f}}(z)
$$

In particular, we denote $T_{\mathbb{R}^{2}} f$ by $\left(\partial_{k}^{-1} f\right)(k)(k \in \mathbb{C})$.

Lemma 2.1. Let $p>2$ and $\alpha=1-2 / p$. Then $T$ and $\bar{T}$ are bounded operators from $L^{p}(\Omega)$ to $C^{\alpha}(\bar{\Omega})$ and satisfy

$$
\|T f\|_{C^{\alpha}(\Omega)},\|\bar{T} f\|_{C^{\alpha}(\Omega)} \leq C_{1}\|f\|_{L^{p}(\Omega)}, \quad \forall f \in L^{p}(\Omega),
$$

where $C_{1}$ is a positive constant which depends only on $p$ and $\Omega$.

Lemma 2.2. For $f \in L^{1}(\Omega)$, we have the estimate

$$
\left\|\partial_{z} T f\right\|_{L^{p}(\Omega)} \leq C_{2}\|f\|_{L^{p}(\Omega)}, \quad \forall f \in L^{p}(\Omega),
$$

where $C_{2}$ is a positive constant which depends only on $p$ and $\Omega$. Similarly for $\bar{T}$.

Lemmas 2.1 and 2.2 are proved in Vekua [29, Chapter 1].

Lemma 2.3. The map $k \mapsto(\bar{\partial}-i k)^{-1}$ is holomorphic on $\mathbb{C}$ in the strong operator topology $L^{\infty}(\Omega) \rightarrow L^{\infty}(\Omega)$.

This lemma is proved in a manner similar to that of Nachman [22, Lemma $2.2]$ (see also [15]). 


\section{$\S 3$. CGO solutions}

In this section we construct the CGO solutions for the system (1.6) and derive some of their properties.

For $x=\left(x_{1}, x_{2}\right) \in \mathbb{R}^{2}$, we put $z=x_{1}+i x_{2}$. In what follows, we abbreviate functions $u(z, \bar{z})$ as $u(z), \partial_{z}$ as $\partial$, and $\bar{\partial}_{z}$ as $\bar{\partial}$. The equation $(-\Delta+V(x)) u(x)=0$ is rewritten as

$$
(\bar{\partial} \partial-q(z)) u(z)=0, \quad q(z)=\frac{1}{4} V(z) .
$$

Let $u$ be a solution to (3.1). Then it is easy to see that

$$
(\mathcal{D}-\boldsymbol{Q})\left(\begin{array}{c}
\partial u \\
u \\
\bar{\partial} u
\end{array}\right)=\mathbf{0} \quad \text { in } \Omega,
$$

where

$$
\mathcal{D}=\left(\begin{array}{lll}
\bar{\partial} & 0 & 0 \\
0 & \partial & 0 \\
0 & 0 & \partial
\end{array}\right), \quad \boldsymbol{Q}=\left(\begin{array}{lll}
0 & q & 0 \\
1 & 0 & 0 \\
0 & q & 0
\end{array}\right)
$$

Consider the equation

$$
(\mathcal{D}-\boldsymbol{Q}) \Phi=\boldsymbol{O} \quad \text { in } \Omega .
$$

In this section we construct the CGO solutions to (3.2) and derive some of their properties.

\section{§3.1. Existence}

For each $k \in \mathbb{C}$, we seek solutions to (3.2) of the form $\boldsymbol{\Phi}=\boldsymbol{\Phi}(z, k)=\boldsymbol{M} \boldsymbol{E}$, where $\boldsymbol{M}=\boldsymbol{M}(z, k)=\left(m_{i j}(z, k)\right)$ is a $3 \times 3$ matrix and

$$
\boldsymbol{E}=\boldsymbol{E}(z, k)=\left(\begin{array}{ccc}
e^{i k z} & 0 & 0 \\
0 & e^{-i k \bar{z}} & 0 \\
0 & 0 & e^{-i k \bar{z}}
\end{array}\right)
$$

Let $e_{k}=e_{k}(z)=e^{i(k z+\bar{k} \bar{z})}$ and $\mathcal{P}_{k}$ be the operator defined by

$$
\mathcal{P}_{k} \boldsymbol{X}=\left(\begin{array}{ccc}
x_{11} & e_{-\bar{k}} x_{12} & e_{-\bar{k}} x_{13} \\
e_{k} x_{21} & x_{22} & x_{23} \\
e_{k} x_{31} & x_{32} & x_{33}
\end{array}\right) \quad \text { for } \boldsymbol{X}=\left(x_{i j}\right)_{1 \leq i, j \leq 3} .
$$

Then simple computation shows that

$$
\mathcal{D} \mathcal{P}_{k} \boldsymbol{M}-\mathcal{P}_{k} \boldsymbol{Q} \boldsymbol{M}=\boldsymbol{O} \quad \text { in } \Omega .
$$


We will construct $\boldsymbol{M}$. Let $|\Omega|=\int_{\Omega} d x$ and

$$
\mathcal{T}=\left(\begin{array}{ccc}
T & 0 & 0 \\
0 & \bar{T} & 0 \\
0 & 0 & \bar{T}
\end{array}\right), \quad \mathcal{T}_{k}=\mathcal{P}_{-k} \mathcal{T} \mathcal{P}_{k}
$$

The following lemmas require almost the same assumptions. We denote the common part by $(\mathrm{A}-1)$ :

(A-1) $q \in L^{p}(\Omega)$ with $C_{1}^{2}|\Omega|^{1 / p}\|q\|_{L^{p}(\Omega)}<1$ for $p>2$, where $C_{1}$ is the positive constant given in Lemma 2.1 .

Lemma 3.1. Assume $(\mathrm{A}-1)$. Then $\left(\boldsymbol{I}-\mathcal{T}_{k} \boldsymbol{Q}\right)^{-1}$ exists on $C^{\alpha}(\bar{\Omega})$.

Proof. It follows from $q \in L^{p}(\Omega)$ and Lemma 2.1 that $\mathcal{T}_{k} \boldsymbol{Q}$ is a compact operator on $C^{\alpha}(\Omega)$. Therefore, it suffices to show the injectivity of $\boldsymbol{I}-\boldsymbol{T}_{k} \boldsymbol{Q}$. Consider the equation $\left(\boldsymbol{I}-\mathcal{T}_{k} \boldsymbol{Q}\right) \boldsymbol{X}=\boldsymbol{O}$. Then $\boldsymbol{X}=\left(x_{i j}\right)_{1 \leq i, j \leq 3}$ satisfies

$$
\begin{aligned}
& \left\{\begin{array} { l } 
{ x _ { 1 1 } - T ( q x _ { 2 1 } ) = 0 , } \\
{ e _ { k } x _ { 2 1 } - \overline { T } ( e _ { k } x _ { 1 1 } ) = 0 , } \\
{ e _ { k } x _ { 3 1 } - \overline { T } ( e _ { k } q x _ { 2 1 } ) = 0 , }
\end{array} \left\{\begin{array}{l}
e_{-\bar{k}} x_{12}-T\left(e_{-\bar{k}} q x_{22}\right)=0, \\
x_{22}-\bar{T} x_{12}=0, \\
x_{32}-\bar{T}\left(q x_{22}\right)=0,
\end{array}\right.\right. \\
& \left\{\begin{array}{l}
e_{-\bar{k}} x_{13}-T\left(e_{-\bar{k}} q x_{23}\right)=0, \\
x_{23}-\bar{T} x_{13}=0, \\
x_{33}-\bar{T}\left(q x_{23}\right)=0 .
\end{array}\right.
\end{aligned}
$$

Since $C_{1}^{2}|\Omega|^{1 / p}\|q\|_{L^{p}(\Omega)}<1$, it follows that $x_{i j}=0,1 \leq i, j \leq 2$, as shown in Kang-Uhlmann [15, Lemma 2]. Using Lemma 2.1, we have

$$
\begin{aligned}
& \left\|x_{23}\right\|_{C^{\alpha}(\Omega)}=\left\|\bar{T} x_{13}\right\|_{C^{\alpha}(\Omega)} \leq C_{1}\left\|x_{13}\right\|_{L^{p}(\Omega)} \leq C_{1}|\Omega|^{1 / p}\left\|x_{13}\right\|_{C^{\alpha}(\Omega)} \\
& \left\|x_{13}\right\|_{C^{\alpha}(\Omega)} \leq C_{1}\left\|e_{-\bar{k}} q x_{23}\right\|_{L^{p}(\Omega)} \leq C_{1}\|q\|_{L^{p}(\Omega)}\left\|x_{23}\right\|_{C^{\alpha}(\Omega)}
\end{aligned}
$$

Now $C_{1}^{2}|\Omega|^{1 / p}\|q\|_{L^{p}(\Omega)}<1$ together with these estimates implies that $x_{13}=$ $x_{23}=0$, which allows us to obtain $x_{31}=x_{32}=x_{33}=0$. Thus $\boldsymbol{X}=\boldsymbol{O}$ and Lemma 3.1 is proved.

Now we define $\boldsymbol{M}=\boldsymbol{M}(\cdot, k) \in C^{\alpha}(\bar{\Omega})$ by

$$
\boldsymbol{M}=\left(\boldsymbol{I}-\mathcal{T}_{k} \boldsymbol{Q}\right)^{-1} \boldsymbol{I} .
$$

It is easy to see that $\boldsymbol{M}$ satisfies (3.3). The function $\boldsymbol{\Phi}=\boldsymbol{M} \boldsymbol{E}$ is called the $C G O$ solution to the equation (3.2). 
§3.2. Asymptotic behavior of $\boldsymbol{M}(z, k)$ as $|k| \rightarrow \infty$

In this subsection, it will be shown that

$$
\boldsymbol{M}(z, k) \rightarrow\left(\begin{array}{ccc}
1 & 0 & 0 \\
0 & 1 & 0 \\
0 & \bar{T} q & 1
\end{array}\right), \quad|k| \rightarrow \infty .
$$

From this property together with Lemma 2.2 we obtain the formula

$$
q(z)=\partial \lim _{|k| \rightarrow \infty} m_{32}(z, k) .
$$

Lemma 3.2. Assume (A-1) and let $\boldsymbol{M}=\left(m_{i j}(z, k)\right)_{1 \leq i, j \leq 3}$ be the function defined by (3.4). Then

$$
\begin{aligned}
& \left\|m_{11}(\cdot, k)-1\right\|_{L^{\infty}(\Omega)}+\left\|m_{22}(\cdot, k)-1\right\|_{L^{\infty}(\Omega)} \leq \frac{2 C_{1}}{|k|}\|q\|_{L^{p}(\Omega)}, \\
& \left\|m_{12}(\cdot, k)\right\|_{L^{\infty}(\Omega)}+\left\|m_{21}(\cdot, k)\right\|_{L^{\infty}(\Omega)} \leq \frac{2}{|k|}, \\
& m_{13}(z, k)=m_{23}(z, k)=0, \quad m_{33}(z, k)=1, \quad \forall z \in \Omega \\
& \left\|m_{31}(\cdot, k)\right\|_{L^{\infty}(\Omega)} \leq \frac{2 C_{1}}{|k|}\|q\|_{L^{p}(\Omega)}, \\
& \left\|m_{32}(\cdot, k)-(\bar{T} q)(\cdot)\right\|_{L^{\infty}(\Omega)} \leq \frac{2 C_{1}^{2}}{|k|}\|q\|_{L^{p}(\Omega)}^{2}
\end{aligned}
$$

for $k \in \mathbb{C}$ such that $2\left\{\left(1+\frac{C_{3}}{2 \pi}\right)+C_{2}\right\} C_{1}\|q\|_{L^{p}(\Omega)}<|k|$, where $C_{2}$ is the positive constant given in Lemma 2.2 .

Proof. The estimates (3.5) and (3.6) are proved in [15, Theorem 5]. To prove the identities $(3.7)$ we first note that $m_{i 3}(i=1,2,3)$ satisfy

$$
\left\{\begin{array}{l}
m_{33}-\bar{T}\left(q m_{23}\right)=1 \\
m_{13}-e_{\bar{k}} T\left(e_{-\bar{k}} q m_{23}\right)=0 \\
m_{23}-\bar{T} m_{13}=0
\end{array}\right.
$$

From the generalized Cauchy integral formula and Lemma 2.1 we have

$$
\begin{aligned}
& \left\|m_{23}(\cdot, k)\right\|_{L^{\infty}(\Omega)}=\left\|\bar{T}\left(m_{13}\right)\right\|_{L^{\infty}(\Omega)} \\
\leq & \frac{1}{|k|}\left\{\left\|\bar{T}\left(\partial m_{13}\right)\right\|_{L^{\infty}(\Omega)}+\left\|\bar{T}\left(e_{\bar{k}} \partial\left(e_{-\bar{k}} m_{13}\right)\right)\right\|_{L^{\infty}(\Omega)}\right\} \\
\leq & \frac{1}{|k|}\left\{\left\|m_{13}\right\|_{L^{\infty}(\Omega)}+\left\|\frac{1}{2 \pi i} \int_{\partial \Omega} \frac{m_{13}(\zeta, k)}{\zeta-z} d \bar{\zeta}\right\|_{L^{\infty}(\Omega)}+C_{1}\left\|\partial\left(e_{-\bar{k}} m_{13}\right)\right\|_{L^{p}(\Omega)}\right\} \\
\leq & \frac{1}{|k|}\left\{\left(1+\frac{C_{3}}{2 \pi}\right)\left\|m_{13}(\cdot, k)\right\|_{L^{\infty}(\Omega)}+C_{1}\left\|\partial\left(e_{-\bar{k}} m_{13}\right)\right\|_{L^{p}(\Omega)}\right\},
\end{aligned}
$$


where $C_{3}$ is a positive constant independent of $k$. According to Lemma 2.2, the second term on the right-hand side in (3.10) is estimated as follows:

$$
\begin{aligned}
\left\|\partial\left(e_{-\bar{k}} m_{13}\right)\right\|_{L^{p}(\Omega)} & =\left\|\partial T\left(e_{-\bar{k}} q m_{23}\right)\right\|_{L^{p}(\Omega)} \leq C_{2}\left\|q m_{23}\right\|_{L^{p}(\Omega)} \\
& \leq C_{2}\|q\|_{L^{p}(\Omega)}\left\|m_{23}\right\|_{L^{\infty}(\Omega)} .
\end{aligned}
$$

This estimate together with (3.10) shows that

$$
\left\|m_{23}(\cdot, k)\right\|_{L^{\infty}(\Omega)} \leq \frac{1}{|k|}\left\{\left(1+\frac{C_{3}}{2 \pi}\right)\left\|m_{13}\right\|_{L^{\infty}(\Omega)}+C_{1} C_{2}\|q\|_{L^{p}(\Omega)}\left\|m_{23}\right\|_{L^{\infty}(\Omega)}\right\} .
$$

Using the estimate

$$
\begin{aligned}
\left\|m_{13}(\cdot, k)\right\|_{L^{\infty}(\Omega)} & =\left\|T\left(e_{-\bar{k}} q m_{23}\right)\right\|_{L^{\infty}(\Omega)} \leq C_{1}\left\|e_{-\bar{k}} q m_{23}\right\|_{L^{p}(\Omega)} \\
& \leq C_{1}\|q\|_{L^{p}(\Omega)}\left\|m_{23}(\cdot, k)\right\|_{L^{\infty}(\Omega)}
\end{aligned}
$$

we obtain

$$
\left\|m_{23}(\cdot, k)\right\|_{L^{\infty}(\Omega)} \leq \frac{1}{|k|}\left\{\left(1+\frac{C_{3}}{2 \pi}\right)+C_{2}\right\} C_{1}\|q\|_{L^{p}(\Omega)}\left\|m_{23}(\cdot, k)\right\|_{L^{\infty}(\Omega)} .
$$

Thus $\left\|m_{23}(\cdot, k)\right\|_{L^{\infty}(\Omega)}=0$ for $k \in \mathbb{C}$ satisfying $\left\{\left(1+\frac{C_{3}}{2 \pi}\right)+C_{2}\right\} C_{1}\|q\|_{L^{p}(\Omega)}<|k|$. From (3.11) we get $\left\|m_{13}(\cdot, k)\right\|_{L^{\infty}(\Omega)}=0$. Moreover, the estimate

$$
\begin{aligned}
\left\|m_{33}(\cdot, k)-1\right\|_{C^{\alpha}(\Omega)} & =\left\|\bar{T}\left(q m_{23}\right)\right\|_{C^{\alpha}(\Omega)} \\
& \leq C_{1}\left\|q m_{23}\right\|_{L^{p}(\Omega)} \leq C_{1}\|q\|_{L^{p}(\Omega)}\left\|m_{23}(\cdot, k)\right\|_{L^{\infty}(\Omega)}
\end{aligned}
$$

yields $\left\|m_{33}(\cdot, k)-1\right\|_{L^{\infty}(\Omega)}=0$. Thus the identities (3.7) hold.

Next, noting that $m_{3 j}(j=1,2)$ satisfy

$$
\left\{\begin{array}{l}
m_{31}-e_{-k} \bar{T}\left(e_{k} q m_{21}\right)=0, \\
m_{32}-\bar{T}\left(q m_{22}\right)=0,
\end{array}\right.
$$

and using (3.5) and (3.6), we get the estimates (3.8) and (3.9):

$$
\begin{aligned}
\left\|m_{31}(\cdot, k)\right\|_{L^{\infty}(\Omega)} & =\left\|\bar{T}\left(e_{k} q m_{21}\right)\right\|_{L^{\infty}(\Omega)} \leq C_{1}\left\|q m_{21}(\cdot, k)\right\|_{L^{p}(\Omega)} \\
& \leq C_{1}\|q\|_{L^{p}(\Omega)}\left\|m_{21}(\cdot, k)\right\|_{L^{\infty}(\Omega)} \leq \frac{2 C_{1}}{|k|}\|q\|_{L^{p}(\Omega)},
\end{aligned}
$$

and

$$
\begin{aligned}
\left\|m_{32}(\cdot, k)-\bar{T} q(\cdot)\right\|_{L^{\infty}(\Omega)} & =\left\|\bar{T}\left(q m_{22}\right)-\bar{T} q\right\|_{L^{\infty}(\Omega)}=\left\|\bar{T} q\left(m_{22}-1\right)\right\|_{L^{\infty}(\Omega)} \\
& \leq C_{1}\|q\|_{L^{p}(\Omega)}\left\|m_{22}(\cdot, k)-1\right\|_{L^{\infty}(\Omega)} \leq \frac{2 C_{1}^{2}}{|k|}\|q\|_{L^{p}(\Omega)}^{2} .
\end{aligned}
$$

Lemma 3.2 is proved. 


\section{$\S 3.3 . \bar{\partial}$-equation}

In this subsection, we derive another property of $\boldsymbol{M}(z, k)$ defined by (3.4). We need some notations. For a $3 \times 3$ matrix $\boldsymbol{X}=\left(x_{i j}\right)$, we denote a submatrix by $[\boldsymbol{X}]_{2}=\left(x_{i j}\right)_{1 \leq i, j \leq 2}$. We first recall that $[\boldsymbol{M}]_{2}=\left[\left(\boldsymbol{I}-\mathcal{T}_{k} \boldsymbol{Q}\right)^{-1} \boldsymbol{I}\right]_{2}$ satisfies the system of differential equations with respect to $k \in \mathbb{C}$, which is called the $\bar{\partial}$ equation.

Lemma 3.3 (Kang and Uhlmann [15]). Assume (A-1). Let $\boldsymbol{\Gamma}_{z}(k)$ be the function given in Step 3 and

$$
\boldsymbol{S}(k)=\left(\begin{array}{cc}
0 & \frac{1}{2 \pi i} \int_{\partial \Omega} e_{-\bar{k}}(\zeta) m_{12}(\zeta, k) d \zeta \\
-\frac{1}{2 \pi i} \int_{\partial \Omega} e_{k}(\zeta) m_{21}(\zeta, k) d \bar{\zeta} & 0
\end{array}\right) .
$$

Then

$$
\bar{\partial}_{k}[\boldsymbol{M}(z, k)]_{2}=[\boldsymbol{M}(z, \bar{k})]_{2} \boldsymbol{\Gamma}_{z}(k) \boldsymbol{S}(k) \text { in } L^{\infty}(\Omega) .
$$

Note that substituting the identity $\boldsymbol{\Phi}=\boldsymbol{M} \boldsymbol{E}$ into $\boldsymbol{S}(k)$ gives

$$
\boldsymbol{S}(k)=\left(\begin{array}{cc}
0 & \frac{1}{2 \pi i} \int_{\partial \Omega} e^{-i \bar{k} \zeta} \phi_{12}(\zeta, k) d \zeta \\
-\frac{1}{2 \pi i} \int_{\partial \Omega} e^{i \bar{k} \bar{\zeta}} \phi_{21}(\zeta, k) d \bar{\zeta} & 0
\end{array}\right) .
$$

This identity means that $\boldsymbol{S}(k)$ can be determined from the boundary values of the CGO solution, $\left.\phi_{12}\right|_{\partial \Omega}$ and $\left.\phi_{21}\right|_{\partial \Omega}$.

In the previous subsection, we proved that $q(z)$ can be calculated from $m_{32}(z, k)$. Hence we are interested in the properties of $m_{32}(z, k)$. We will show that $m_{31}(z, k)$ and $m_{32}(z, k)$ also satisfy a $\bar{\partial}$ type equation.

Lemma 3.4. Assume (A-1) and let $\boldsymbol{M}=\left(m_{i j}(z, k)\right)_{1 \leq i, j \leq 3}$ be the function defined by (3.4). Then

$$
\left\{\begin{array}{l}
\bar{\partial}_{k} m_{31}(z, k)=-i e_{-k}(z) s_{31}(k)+i e_{-k}(z) s_{21}(k) m_{32}(z, \bar{k}), \\
\bar{\partial}_{k} m_{32}(z, k)=i e_{\bar{k}}(z) s_{12}(k) m_{31}(z, \bar{k})
\end{array}\right.
$$

where

$$
\begin{aligned}
& s_{12}(k)=-\frac{1}{\pi} \int_{\Omega} e_{-\bar{k}}(\zeta) q(\zeta) m_{22}(\zeta, k) d \zeta_{R} d \zeta_{I}, \\
& s_{21}(k)=\frac{1}{\pi} \int_{\Omega} e_{k}(\zeta) m_{11}(\zeta, k) d \zeta_{R} d \zeta_{I}, \\
& s_{31}(k)=\frac{1}{\pi} \int_{\Omega} e_{k}(\zeta) q(\zeta) m_{21}(\zeta, k) d \zeta_{R} d \zeta_{I} .
\end{aligned}
$$


Proof. We first prove the identity

$$
\bar{\partial}_{k}\left(\bar{T} q m_{22}\right)=\bar{T}\left(q \bar{\partial}_{k} m_{22}\right)
$$

Since $\bar{T}$ is a bounded operator $L^{p}(\Omega) \rightarrow C^{\alpha}(\Omega)$ and $q$ is in $L^{p}(\Omega)$, it suffices to show the uniform boundedness of $\bar{\partial}_{k} m_{22}$ by the application of dominated convergence. Since $m_{i j}, 1 \leq i, j \leq 2$, satisfy

$$
\left\{\begin{array} { l } 
{ m _ { 1 1 } - T ( q m _ { 2 1 } ) = 1 , } \\
{ m _ { 2 1 } - e _ { - k } \overline { T } ( e _ { k } m _ { 1 1 } ) = 0 , }
\end{array} \quad \left\{\begin{array}{l}
m_{12}-e_{\bar{k}} T\left(e_{-\bar{k}} q m_{22}\right)=0, \\
m_{22}-\bar{T}\left(m_{12}\right)=1,
\end{array}\right.\right.
$$

Lemma 2.1 yields the estimates

$\left\|m_{22}-1\right\|_{L^{\infty}(\Omega)} \leq C_{1}|\Omega|^{1 / p}\left\|m_{12}\right\|_{L^{\infty}(\Omega)}, \quad\left\|m_{12}\right\|_{L^{\infty}(\Omega)} \leq C_{1}\|q\|_{L^{p}(\Omega)}\left\|m_{22}\right\|_{L^{\infty}(\Omega)}$, $\left\|m_{11}-1\right\|_{L^{\infty}(\Omega)} \leq C_{1}\|q\|_{L^{p}(\Omega)}\left\|m_{21}\right\|_{L^{\infty}(\Omega)}, \quad\left\|m_{21}\right\|_{L^{\infty}(\Omega)} \leq C_{1}|\Omega|^{1 / p}\left\|m_{11}\right\|_{L^{\infty}(\Omega)}$.

If $C_{1}^{2}|\Omega|^{1 / p}\|q\|_{L^{p}(\Omega)}<\delta<1$, then we obtain

$$
\begin{aligned}
& \left\|m_{11}\right\|_{L^{\infty}(\Omega)},\left\|m_{22}\right\|_{L^{\infty}(\Omega)} \leq \frac{1}{1-\delta} \\
& \left\|m_{12}\right\|_{L^{\infty}(\Omega)} \leq \frac{C_{1}\|q\|_{L^{p}(\Omega)}}{1-\delta}, \quad\left\|m_{21}\right\|_{L^{\infty}(\Omega)} \leq \frac{C_{1}|\Omega|^{1 / p}}{1-\delta}
\end{aligned}
$$

By Lemma 3.3 we already know that $\bar{\partial}_{k} m_{22}=i s_{12}(k) e_{\bar{k}}(z) m_{21}(z, \bar{k})$. Hence we obtain the boundedness of $\bar{\partial}_{k} m_{22}$ :

$$
\begin{aligned}
\left|\bar{\partial}_{k} m_{22}\right| & \leq \frac{C_{1}|\Omega|^{1 / p}}{1-\delta}\left|s_{12}(k)\right| \leq \frac{1}{\pi} \frac{C_{1}|\Omega|^{1 / p}}{1-\delta} \int_{\Omega}\left|q(z) m_{22}(z, k)\right| d z_{R} d z_{I} \\
& \leq \frac{1}{\pi} \frac{C_{1}|\Omega|}{(1-\delta)^{2}}\|q\|_{L^{p}(\Omega)} .
\end{aligned}
$$

Using Lemma 3.3 and the identities (3.12) and (3.16), we have

$$
\begin{aligned}
\bar{\partial}_{k} m_{32}(z, k) & =\bar{\partial}_{k}\left(\bar{T} q m_{22}\right)=\bar{T}\left(q \bar{\partial}_{k} m_{22}\right) \\
& =i s_{12}(k) \bar{T}\left(q e_{\bar{k}} m_{21}(\cdot, \bar{k})\right)=i s_{12}(k) e_{\bar{k}}(z) m_{31}(z, \bar{k}) .
\end{aligned}
$$

Similarly, using the identity

$$
\begin{aligned}
\bar{T}\left(e_{k} q \bar{\partial}_{k} m_{21}\right) & =\bar{T}\left(e_{k} q i s_{21}(k) e_{-k} m_{22}(\cdot, \bar{k})\right)=i s_{21}(k) \bar{T}\left(q m_{22}(\cdot, \bar{k})\right) \\
& =i s_{21}(k) m_{32}(z, \bar{k})
\end{aligned}
$$


together with Lemma 2.3, we obtain the equation

$$
\begin{aligned}
\bar{\partial}_{k} m_{31}(z, k) & =\bar{\partial}_{k}\left(e_{-k} \bar{T}\left(e_{k} q m_{21}\right)\right) \\
& =\bar{\partial}_{k}\left(-\frac{1}{\pi} \int_{\Omega} \frac{e_{k}(\zeta-z)}{\bar{\zeta}-\bar{z}} q(\zeta) m_{21}(\zeta, k) d \zeta_{R} d \zeta_{I}\right) \\
& =-\frac{1}{\pi}\left(i \int_{\Omega} e_{k}(\zeta-z) q(\zeta) m_{21}(\zeta, k) d \zeta_{R} d \zeta_{I}\right. \\
& \left.\quad+\int_{\Omega} \frac{e_{k}(\zeta-z)}{\bar{\zeta}-\bar{z}} q(\zeta) \bar{\partial}_{k} m_{21}(\zeta, k) d \zeta_{R} d \zeta_{I}\right) \\
& =-i e_{-k}(z) s_{31}(k)+e_{-k}(z) \bar{T}\left(e_{k} q \bar{\partial}_{k} m_{21}\right) \\
& =-i e_{-k}(z) s_{31}(k)+i e_{-k}(z) s_{21}(k) m_{32}(z, \bar{k}) .
\end{aligned}
$$

Thus, Lemma 3.4 is proved.

By Green's formula,

$$
\begin{aligned}
& s_{12}(k)=-\frac{1}{\pi} \int_{\Omega} e_{-\bar{k}}(\zeta) q(\zeta) m_{22}(\zeta, k) d \zeta_{R} d \zeta_{I}=\frac{1}{2 \pi i} \int_{\partial \Omega} e^{-i \bar{k} \zeta} \phi_{12}(\zeta, k) d \zeta \\
& s_{21}(k)=\frac{1}{\pi} \int_{\Omega} e_{k}(\zeta) m_{11}(\zeta, k) d \zeta_{R} d \zeta_{I}=-\frac{1}{2 \pi i} \int_{\partial \Omega} e^{i \bar{k} \bar{\zeta}} \phi_{21}(\zeta, k) d \bar{\zeta}
\end{aligned}
$$

which shows that $s_{12}(k)$ and $s_{21}(k)$ can be determined from the boundary values of the CGO solution, $\left.\phi_{12}\right|_{\partial \Omega}$ and $\left.\phi_{21}\right|_{\partial \Omega}$. We can also determine $s_{31}(k)$ from the boundary values $\left.\phi_{11}\right|_{\partial \Omega}$ and $\left.\phi_{21}\right|_{\partial \Omega}$. Indeed, it follows from (3.17) and Green's formula that

$$
\begin{aligned}
s_{31}(k) & =\frac{1}{\pi} \int_{\Omega}\left\{\bar{\partial}\left(e_{k} m_{11}\right)-m_{11} \bar{\partial} e_{k}\right\} d z_{R} d z_{I} \\
& =\frac{1}{\pi} \int_{\Omega} \bar{\partial}\left(e_{k} m_{11}\right) d z_{R} d z_{I}-\frac{1}{\pi} \int_{\Omega} i \bar{k} e_{k} m_{11} d z_{R} d z_{I} \\
& =\frac{1}{\pi} \int_{\Omega} \bar{\partial}\left(e_{k} m_{11}\right) d z_{R} d z_{I}-\frac{i \bar{k}}{\pi} \int_{\Omega} \partial\left(e_{k} m_{21}\right) d z_{R} d z_{I} \\
& =\frac{1}{2 \pi i} \int_{\partial \Omega} e_{k}(z) m_{11}(z, k) d z+\frac{\bar{k}}{2 \pi} \int_{\partial \Omega} e_{k}(z) m_{21}(z, k) d \bar{z} \\
& =\frac{1}{2 \pi i} \int_{\partial \Omega} e^{i \bar{k} \bar{z}} \Phi_{11}(z, k) d z-i \bar{k} s_{21}(k) .
\end{aligned}
$$

\section{§4. From $\Lambda_{V}$ to $\left.\boldsymbol{\Phi}\right|_{\partial \Omega}$}

In this section we show that the boundary value $\left.\boldsymbol{\Phi}\right|_{\partial \Omega}$ of the CGO solution can be constructed from the DN map $\Lambda_{V}$. Let us denote column vectors by boldface, 
e.g., $\boldsymbol{f}={ }^{t}\left(f_{1}, f_{2}, f_{3}\right)$. We define a set of Cauchy data by

$$
\mathbb{D}_{q}=\left\{\left.\boldsymbol{v}\right|_{\partial \Omega}: \boldsymbol{v} \in C^{\alpha}(\bar{\Omega}) \times C^{1, \alpha}(\bar{\Omega}) \times C^{\alpha}(\bar{\Omega}),(\mathcal{D}-\boldsymbol{Q}) \boldsymbol{v}=\mathbf{0} \text { in } \Omega\right\} .
$$

Let $\mathcal{H}_{V}=\Lambda_{V} \mathcal{L}$ and define a set of functions on $\partial \Omega$ by

$$
\begin{aligned}
\mathbb{B}=\left\{\boldsymbol{h} \in C^{\alpha}(\partial \Omega) \times C^{1, \alpha}(\partial \Omega) \times C^{\alpha}(\partial \Omega):\left(\eta h_{1}-\bar{\eta} h_{3}\right) \in C_{0}^{1, \alpha}(\partial \Omega),\right. \\
\left.i \mathcal{H}_{V}\left(\eta h_{1}-\bar{\eta} h_{3}\right)=\eta h_{1}+\bar{\eta} h_{3}, \tau \cdot \nabla h_{2}=i\left(\eta h_{1}-\bar{\eta} h_{3}\right)\right\},
\end{aligned}
$$

where $C_{0}^{1, \alpha}(\partial \Omega)$ denotes the set of $C^{1, \alpha}$ functions on $\partial \Omega$ such that $\int_{\partial \Omega} f d \sigma=0$ ( $d \sigma$ is the Euclidean surface measure on $\partial \Omega$ ).

The following characterization of the Cauchy data for the first order $\bar{\partial}$-system is shown in a manner similar to that of Knudsen and Tamasan [17].

Lemma 4.1. Let $q \in L^{p}(\Omega)$. Assume that

$$
\boldsymbol{Q}=\left(\begin{array}{lll}
0 & q & 0 \\
1 & 0 & 0 \\
0 & q & 0
\end{array}\right)
$$

Then $\mathbb{D}_{q}=\mathbb{B}$.

Proof. We first show that $\mathbb{B} \subset \mathbb{D}_{q}$. Let $\boldsymbol{h} \in \mathbb{B}$ and $u$ be a $C^{1, \alpha}(\bar{\Omega})$ solution of

$$
\left\{\begin{array}{l}
-\Delta u+V u=0 \quad \text { in } \Omega, \\
\left.u\right|_{\partial \Omega}=i \mathcal{L}\left(\eta h_{1}-\bar{\eta} h_{3}\right) \in C^{1, \alpha}(\partial \Omega) .
\end{array}\right.
$$

Putting ${ }^{t}(\varphi, \psi, \chi)={ }^{t}(\partial u, u, \bar{\partial} u) \in C^{\alpha}(\bar{\Omega}) \times C^{1, \alpha}(\bar{\Omega}) \times C^{\alpha}(\bar{\Omega})$, we deduce that $(\mathcal{D}-\boldsymbol{Q})^{t}(\varphi, \psi, \chi)=\mathbf{0}$. Since $\boldsymbol{h} \in \mathbb{B}$, we obtain the relation

$$
\begin{aligned}
\left.\left(\begin{array}{c}
\varphi \\
\chi
\end{array}\right)\right|_{\partial \Omega} & =\frac{1}{2}\left(\begin{array}{cc}
\bar{\eta} & -i \bar{\eta} \\
\eta & i \eta
\end{array}\right)\left(\begin{array}{c}
\Lambda_{V}\left(\left.u\right|_{\partial \Omega}\right) \\
\tau \cdot \nabla\left(\left.u\right|_{\partial \Omega}\right)
\end{array}\right)=\frac{1}{2}\left(\begin{array}{cc}
\bar{\eta} & -i \bar{\eta} \\
\eta & i \eta
\end{array}\right)\left(\begin{array}{c}
i \mathcal{H}_{V}\left(\eta h_{1}-\bar{\eta} h_{3}\right) \\
i\left(\eta h_{1}-\bar{\eta} h_{3}\right)
\end{array}\right) \\
& =\frac{1}{2}\left(\begin{array}{cc}
\bar{\eta} & -i \bar{\eta} \\
\eta & i \eta
\end{array}\right)\left(\begin{array}{c}
\eta h_{1}+\bar{\eta} h_{3} \\
i\left(\eta h_{1}-\bar{\eta} h_{3}\right)
\end{array}\right)=\left(\begin{array}{l}
h_{1} \\
h_{3}
\end{array}\right) .
\end{aligned}
$$

The identity $\left.\psi\right|_{\partial \Omega}=\left.u\right|_{\partial \Omega} \in C^{1, \alpha}(\partial \Omega)$ implies that $\boldsymbol{h} \in \mathbb{D}_{q}$.

Next we show that $\mathbb{D}_{q} \subset \mathbb{B}$. Let $\boldsymbol{h} \in \mathbb{D}_{q}$ and ${ }^{t}(\varphi, \psi, \chi) \in C^{\alpha}(\bar{\Omega}) \times C^{1, \alpha}(\bar{\Omega}) \times$ $C^{\alpha}(\bar{\Omega})$ be a solution of

$$
\left\{\begin{array}{l}
(\mathcal{D}-\boldsymbol{Q})^{t}(\varphi, \psi, \chi)=\mathbf{0} \quad \text { in } \Omega, \\
\left.{ }^{t}(\varphi, \psi, \chi)\right|_{\partial \Omega}=\boldsymbol{h} .
\end{array}\right.
$$

Simple computation shows that $\varphi, \psi$ and $\chi$ satisfy $\bar{\partial} \varphi=q \psi, \partial \psi=\varphi$ and $\partial \chi=q \psi$. Since there exists a $C^{1, \alpha}(\bar{\Omega})$ function $u$ such that $\psi=u, \varphi=\partial u$ and $\chi=\bar{\partial} u$, we 
obtain the relation

$$
\begin{aligned}
\left(\begin{array}{l}
h_{1} \\
h_{2} \\
h_{3}
\end{array}\right) & =\left.\left(\begin{array}{c}
\varphi \\
\psi \\
\chi
\end{array}\right)\right|_{\partial \Omega}=\left.\left(\begin{array}{c}
\partial u \\
u \\
\bar{\partial} u
\end{array}\right)\right|_{\partial \Omega}=\frac{1}{2}\left(\begin{array}{c}
\bar{\eta} \Lambda_{V} h_{2}-i \bar{\eta} \tau \cdot \nabla h_{2} \\
2 h_{2} \\
\eta \Lambda_{V} h_{2}+i \eta \tau \cdot \nabla h_{2}
\end{array}\right) \\
& =\frac{1}{2}\left(\begin{array}{ccc}
\bar{\eta} & 0 & -i \bar{\eta} \\
0 & 2 & 0 \\
\eta & 0 & i \eta
\end{array}\right)\left(\begin{array}{c}
\Lambda_{V} h_{2} \\
h_{2} \\
\tau \cdot \nabla h_{2}
\end{array}\right) .
\end{aligned}
$$

The identity

$$
\left(\begin{array}{c}
\Lambda_{V} h_{2} \\
h_{2} \\
\tau \cdot \nabla h_{2}
\end{array}\right)=\left(\begin{array}{ccc}
\eta & 0 & \bar{\eta} \\
0 & 1 & 0 \\
i \eta & 0 & -i \bar{\eta}
\end{array}\right)\left(\begin{array}{l}
h_{1} \\
h_{2} \\
h_{3}
\end{array}\right)
$$

implies that $\boldsymbol{h} \in \mathbb{B}$. Lemma 4.1 is proved.

Next we give a characterization of the traces of the CGO solutions.

Lemma 4.2. Assume that $\boldsymbol{Q}$ satisfies the assumption of Lemma 4.1. Then there exist $\boldsymbol{\psi}^{(j)} \in \mathbb{D}_{q}(j=1,2,3)$ such that

$$
\begin{aligned}
& \left.\mathcal{P}_{-k} \mathcal{F}\left(\mathcal{P}_{k} \boldsymbol{\Psi} \boldsymbol{E}^{-1}\right)\right|_{\partial \Omega}=\boldsymbol{I} \\
& \left(\begin{array}{ccc}
i \Lambda_{V} \mathcal{L} & 0 & -i \Lambda_{V} \mathcal{L} \\
0 & i \frac{\partial}{\partial \tau} & 0
\end{array}\right)\left(\begin{array}{ccc}
\eta & 0 & 0 \\
0 & 1 & 0 \\
0 & 0 & \bar{\eta}
\end{array}\right) \boldsymbol{\Psi}=\left(\begin{array}{ccc}
\eta & 0 & \bar{\eta} \\
-\eta & 0 & \bar{\eta}
\end{array}\right) \boldsymbol{\Psi}
\end{aligned}
$$

where $\boldsymbol{\Psi}=\left(\boldsymbol{\psi}^{(j)}\right)_{j=1,2,3}$ is a $3 \times 3$ matrix. Moreover $\boldsymbol{\Psi}$ is the only CGO solution on $\partial \Omega$, i.e., $\boldsymbol{\Psi}=\left.\boldsymbol{\Phi}\right|_{\partial \Omega}$.

Proof. Let $\boldsymbol{\Phi}=\boldsymbol{M} \boldsymbol{E}$ be the CGO solution. Then $(\mathcal{D}-\boldsymbol{Q}) \boldsymbol{\Phi}=\boldsymbol{O}$. By Lemma 4.1 we have $\boldsymbol{\Phi} \in \mathbb{B}$, which shows that $\boldsymbol{\Phi}$ satisfies (4.2). Taking into account that $\boldsymbol{M}$ is a solution to $\mathcal{D} \mathcal{P}_{k} \boldsymbol{M}-\mathcal{P}_{k} \boldsymbol{Q} \boldsymbol{M}=\boldsymbol{O}$, it follows from the generalized Cauchy integral formula that

$$
\boldsymbol{M}-\mathcal{P}_{-k} \mathcal{T} \mathcal{P}_{k} Q \boldsymbol{M}=\mathcal{P}_{-k} \mathcal{F}\left(\left.\mathcal{P}_{k} M\right|_{\partial \Omega}\right)
$$

By (3.4),

$$
\mathcal{P}_{-k} \mathcal{F}\left(\left.\mathcal{P}_{k} \boldsymbol{M}\right|_{\partial \Omega}\right)=\left(\boldsymbol{I}-\mathcal{T}_{k} \boldsymbol{Q}\right) \boldsymbol{M}=\boldsymbol{I},
$$

which implies (4.1).

Now, suppose that $\boldsymbol{\Psi}$ satisfies (4.1) and (4.2). Then $\boldsymbol{\Psi} \in \mathbb{B}$. According to Lemma 4.1 , there exists a $3 \times 3$ matrix $\boldsymbol{H}$ such that

$$
(\mathcal{D}-\boldsymbol{Q}) \boldsymbol{H}=\boldsymbol{O},\left.\quad \boldsymbol{H}\right|_{\partial \Omega}=\boldsymbol{\Psi} .
$$


Let $\boldsymbol{G}=\boldsymbol{H} \boldsymbol{E}^{-1}$. Then $\boldsymbol{G}$ satisfies $\mathcal{P}_{-k} \mathcal{D} \mathcal{P}_{k} \boldsymbol{G}-\boldsymbol{Q} \boldsymbol{G}=\boldsymbol{O}$. The generalized Cauchy integral formula yields

$$
\left(\boldsymbol{I}-\mathcal{T}_{k} \boldsymbol{Q}\right) \boldsymbol{G}=\mathcal{P}_{-k} \mathcal{F}\left(\left.\mathcal{P}_{k} \boldsymbol{\Psi} \boldsymbol{E}^{-1}\right|_{\partial \Omega}\right)
$$

By (4.1), $\boldsymbol{G}=\left(\boldsymbol{I}-\mathcal{T}_{k} \boldsymbol{Q}\right)^{-1} \boldsymbol{I}=\boldsymbol{M}$, which gives

$$
\boldsymbol{\Psi}=\left.\boldsymbol{H}\right|_{\partial \Omega}=\left.\boldsymbol{M E}\right|_{\partial \Omega}=\left.\boldsymbol{\Phi}\right|_{\partial \Omega} .
$$

Lemma 4.2 is proved.

The characterization (4.1) and (4.2) gives a method for finding the CGO solution $\left.\boldsymbol{\Phi}\right|_{\partial \Omega}$ from the DN map $\Lambda_{V}$.

\section{$\S 5$. From $\left.\Phi\right|_{\partial \Omega}$ to $m_{32}$}

In this section we show the unique solvability of the system (3.15). Let $s_{12}(k)$, $s_{21}(k)$ and $s_{31}(k)$ be the functions given in Lemma 3.4 .

Lemma 5.1. Assume (A-1) and suppose that $q \in W^{1, p}(\Omega)$. Then for $k \in \mathbb{C}$,

$$
\begin{aligned}
& \left|s_{12}(k)\right| \leq \frac{\gamma_{1}(q)}{1+|k|}\|q\|_{W^{1, p}(\Omega)}, \quad\left|s_{21}(k)\right| \leq \frac{\gamma^{\prime}}{1+|k|}\left(\|q\|_{L^{p}(\Omega)}+1\right), \\
& \left|s_{31}(k)\right| \leq \frac{\gamma_{2}}{1+|k|}\|q\|_{L^{p}(\Omega)},
\end{aligned}
$$

where $\gamma_{1}(q)$ is a positive constant depending on $\|q\|_{W^{1, p}(\Omega)}$ and uniformly bounded from below, and $\gamma^{\prime}$ and $\gamma_{2}$ are positive constants independent of $|k|$ and $q$.

Proof. The estimates on $s_{12}$ and $s_{21}$ are proved in [15]. Let $C_{1}^{2}|\Omega|^{1 / p}\|q\|_{L^{p}(\Omega)}=$ $\delta<1$. The estimates

$\left\|m_{21}\right\|_{L^{\infty}(\Omega)} \leq C_{1}|\Omega|^{1 / p}\left\|m_{11}\right\|_{L^{\infty}(\Omega)}, \quad\left\|m_{11}\right\|_{L^{\infty}(\Omega)} \leq C_{1}\|q\|_{L^{p}(\Omega)}\left\|m_{21}\right\|_{L^{\infty}(\Omega)}+1$

together with the assumption (A-1) show that for $k \in \mathbb{C}$,

$$
\left\|m_{21}(\cdot, k)\right\|_{L^{\infty}(\Omega)} \leq \frac{C_{1}|\Omega|^{1 / p}}{1-\delta} .
$$

Thus we obtain

$$
\left|s_{31}(k)\right| \leq \frac{C_{1}|\Omega|^{1 / p}}{\pi(1-\delta)}|\Omega|^{1 / p^{\prime}}\|q\|_{L^{p}(\Omega)}=\frac{C_{1}|\Omega|}{\pi(1-\delta)}\|q\|_{L^{p}(\Omega)}
$$

for $k \in \mathbb{C}$. By Lemma 3.2, there exists a positive constant $N$ such that

$$
\left|s_{31}(k)\right| \leq \frac{1}{\pi}\left\|m_{21}\right\|_{L^{\infty}(\Omega)} \int_{\Omega}|q(z)| d z_{R} d z_{I} \leq \frac{2|\Omega|^{1 / p^{\prime}}}{\pi} \frac{1}{|k|}\|q\|_{L^{p}(\Omega)}
$$


for $|k|>N$. Thus we get the estimate

$$
\left|s_{31}(k)\right| \leq \frac{\gamma_{2}}{|k|+1}\|q\|_{L^{p}(\Omega)}, \quad \gamma_{2}=\frac{C_{1}|\Omega|(2+N)}{\pi(1-\delta)}+\frac{2|\Omega|^{1 / p^{\prime}}}{\pi}
$$

for $k \in \mathbb{C}$. Lemma 5.1 is proved.

Let $\boldsymbol{M}=\left(m_{i j}\right)$ be the $3 \times 3$ matrix defined by (3.4). Then $\boldsymbol{m}={ }^{t}\left(m_{31}, m_{32}\right)$ is a solution to the system (3.15). The uniqueness of the solution follows from the next lemma.

Lemma 5.2 (Kang and Uhlmann [15]). Let $d_{1}, d_{2}$ and $\varepsilon$ be positive constants. Assume that

$$
|a(k)| \leq \frac{d_{1}(\varepsilon+1)}{1+|k|}, \quad|b(k)| \leq \frac{d_{2} \varepsilon}{1+|k|} .
$$

If $\varepsilon$ is sufficiently small, then the solution of the system

$$
\left\{\begin{array}{l}
\bar{\partial}_{k} f(k)=a(k) g(\bar{k}) \\
\bar{\partial}_{k} g(k)=b(k) f(\bar{k})
\end{array}\right.
$$

such that $f-1, g \in L^{2, \delta}(\mathbb{C})(-1<\delta<0)$ is unique.

Proof of Theorem 1.3. We have already justified Steps 2, $3^{\prime}$ and $4^{\prime}$ stated in Subsection 1.2. By Lemma 4.2, we obtain the boundary value of the CGO solution from the DN map. The functions $s_{12}(k), s_{21}(k)$ and $s_{31}(k)$ defined in Step $3^{\prime}$ satisfy the estimates given in Lemma 5.1. Therefore we can get the function $m_{32}(z, k)$ on $\Omega \times \mathbb{C}$ by solving the system (3.15). The estimate (3.9) given in Lemma 3.2 shows that $q(z)$ can be obtained from $m_{32}(z, k)$. Thus Theorem 1.3 has been completely proved.

\section{Acknowledgements}

The author wishes to express his sincere gratitude to Professor Hiroshi Isozaki for his helpful comments. The author would also like to thank Professor Masaru Ikehata for information about the paper [3] of Bukhgeim, and the anonymous referee for helpful comments.

This research was partially supported by Grant-in-Aid for Young Scientists (B) (No. 20740078) of Japan Society for the Promotion of Science.

\section{References}

[1] K. Astala and L. Päivärinta, Calderón's inverse conductivity problem in the plane, Ann. of Math. 163 (2006), 265-299. Zbl 1111.35004 MR 2195135 
[2] R. Brown and G. Uhlmann, Uniqueness in the conductivity problem for nonsmooth conductivities in two dimensions, Comm. Partial Differential Equations 22 (1997), 1009-1027. Zbl 0884.35167 MR 1452176

[3] A. L. Bukhgeim, Recovering a potential from Cauchy data in the two-dimensional case, J. Inverse Ill-Posed Problems 16 (2008), 19-33. Zbl 1142.30018 MR 2387648

[4] J. Cheng and M. Yamamoto, Determination of two convection coefficients from Dirichletto-Neumann map in two-dimensional case, SIAM J. Math. Anal. 35 (2003), 1371-1393. Zbl 1061.35164 MR 2083783

[5] E. Francini, Recovering a complex coefficient in a planar domain from the Dirichlet-toNeumann map, Inverse Problems 16 (2000), 107-119. Z Zbl 0968.35125 MR 1741230

[6] P. G. Grinevich, Scattering transformation at fixed non-zero energy for the two-dimensional Schrödinger operator with potential decaying at infinity, Russian Math. Surveys 55 (2000), 1015-1083. Zbl 1022.81057 MR 1840357

[7] M. Ikehata, Mathematics of reconstruction of the surface of discontinuity from observation data - reconstruction formulae for inverse boundary value/source/scattering problems, Tokyo Metropolitan University, Department of Mathematics Seminar Report 2000.

[8] O. Imanuvilov, G. Uhlmann and M. Yamamoto, Global uniqueness from partial Cauchy data in two dimensions, J. Amer. Math. Soc. 23 (2010), 655-691.

[9] V. Isakov, Inverse problems for partial differential equations, 2nd ed., Appl. Math. Sci. 127, Springer, New York, 2006. Zbl 1092.35001 MR 2193218

[10] V. Isakov and A. Nachman, Global uniqueness for a two-dimensional semilinear elliptic inverse problem, Trans. Amer. Math. Soc. 347 (1995), 3375-3390. Zbl 0849.35148 MR 1311909

[11] V. Isakov and Z. Sun, The inverse scattering at fixed energies in two dimensions, Indiana Univ. Math. J. 44 (1995), 883-896. Zbl 0855.35128 MR 1375354

[12] H. Isozaki, H. Nakazawa and G. Uhlmann, Inverse scattering problem in nuclear physicsOptical model, J. Math. Phys. 45 (2004), 2613-2632. Z Zbl 1071.81107 MR 2067577

[13] M. Kadowaki, H. Nakazawa and K. Watanabe, On scattering for wave equations with dissipative terms in layered media, Electron. J. Differential Equations 2011, no. 65, 18 pp. Zbl 1226.35060 MR 2801250

[14] H. Kang, A uniqueness theorem for an inverse boundary value problem in two dimensions, J. Math. Anal. Appl. 270 (2002), 291-302. Zbl 1040.35137 MR 1911766

[15] H. Kang and G. Uhlmann, Inverse problems for the Pauli Hamiltonian in two dimensions, J. Fourier Anal. Appl. 10 (2004), 201-215. Zbl 1081.35141 MR 2054308

[16] K. Knudsen, On the inverse conductivity problem, Ph.D. thesis, Dept. Math. Sci., Aalborg Univ., 2002.

[17] K. Knudsen and A. Tamasan, Reconstruction of less regular conductivities in plane, Comm. Partial Differential Equations 29 (2004), 361-381. Z Zbl 1063.35149 MR 2041600

[18] K. Mochizuki, Scattering theory for wave equations with dissipative term, Publ. RIMS Kyoto Univ. 12 (1976), 383-390. Zbl 0357.35067 MR 0437955

[19] _ Eigenfunction expansions associated with the Schrödinger operator with a complex potential and the scattering inverse problem, Proc. Japan Acad. 43 (1967), 638-643. Zbl 0157.21701 MR 0222494

[20] _ Inverse scattering for a small nonselfadjoint perturbation of the wave equation, in Analysis and applications-ISAAC 2001 (Berlin), Int. Soc. Anal. Appl. Comput. 10, Kluwer, Dordrecht, 2003, 303-316. Zbl 1080.35553 MR 2022755

[21] A. Nachman, Inverse scattering at fixed energy, in Mathematical physics, X (Leipzig, 1991), K. Schmüdgen (ed.), Springer, 1992, 434-441. Zbl 0947.81562 MR 1386440 
[22] Global uniqueness for a two-dimensional inverse boundary value problem, Ann. of Math. 143 (1996), 71-96. Zbl 0857.35135 MR 1370758

[23] Reconstructions from boundary measurements, Ann. of Math. 128 (1988), 531-576. Zbl 0675.35084 MR 0970610

[24] H. Nakazawa, The principle of limiting absorption for the non-selfadjoint Schrödinger operator with energy dependent potential, Tokyo J. Math. 23 (2000), 519-536. Zbl 0984.35124

[25] On wave equations with dissipations, in Analytical methods of analysis and differential equations (AMADE-2006), Vol. 3, Differential Equations, Inst. Math., NAS of Belarus, Minsk, 2006, 102-110.

[26] R. G. Novikov, A multidimensional inverse spectral problem for the equation $-\Delta \psi+(v(x)-$ $E u(x)) \psi=0$, Funct. Anal. Appl. 22 (1988), 263-272. Zbl 0689.35098 MR 0976992

[27] The inverse scattering problem on a fixed energy level for the two-dimensional Schrödinger operator, J. Funct. Anal. 103 (1992), 409-463. Zbl 0762.35077 MR 1151554

[28] J. Sylvester and G. Uhlmann, A global uniqueness theorem for an inverse boundary value problem, Ann. of Math. 125 (1987), 153-169. Zbl 0625.35078 MR 0873380

[29] I. N. Vekua, Generalized analytic functions, Pergamon Press, London, 1962. Z Zbl 0100.07603 MR 0150320

[30] M. Watanabe, An inverse scattering problem for the wave equation with the friction term in two dimensions, in Nonlinear dispersive equations, GAKUTO Int. Ser. Math. Sci. Appl. 26, Gakkōtosho, Tokyo, 2006, 213-226. Zbl 1188.35123 MR 2409134 